\title{
COGNITIVE PERFORMANCE IN MULTIPLE SYSTEM ATROPHY
}

\author{
by T. W. ROBbins, ${ }^{1}$ M. JAMES, ${ }^{2}$ K. W. LANGE, ${ }^{3}$ A. M. OWEN, \\ N. P. QUIN ${ }^{3}$ and C. D. MARSDEN ${ }^{3}$ \\ (From the 'Department of Experimental Psychology, University of Cambridge, the ${ }^{2}$ Department of \\ Psychology, The National Hospital and the ${ }^{3}$ Department of Clinical Neurology, Institute of \\ Neurology, London, UK)
}

\begin{abstract}
SUMMARY
The cognitive performance of a group of patients with multiple system atrophy (MSA) of striato-nigral predominance was compared with that of age and IQ matched control subjects, using three tests sensitive to frontal lobe dysfunction and a battery sensitive to memory and learning deficits in Parkinson's disease and dementia of the Alzheimer type. The MSA group showed significant deficits in all three of the tests previously shown to be sensitive to frontal lobe dysfunction. Thus, a significant proportion of patients from the MSA group failed an attentional set-shifting test, specifically at the stage when an extra-dimensional shift was required. They were also impaired in a subject-ordered test of spatial working memory. The MSA group showed deficits mostly confined to measures of speed of thinking, rather than accuracy, on the Tower of London task. These deficits were seen in the absence of consistent impairments in language or visual perception. Moreover, the MSA group showed no significant deficits in tests of spatial and pattern recognition previously shown to be sensitive to patients early in the course of probable Alzheimer's disease and only a few patients exhibited impairment on the Warrington Recognition Memory Test. There were impairments on other tests of visual memory and learning relative to matched controls, but these could not easily be related to fundamental deficits of memory or learning. Thus, on a matching-to-sample task the patients were impaired at simultaneous but not delayed matching to sample, whereas difficulties in a pattern-location learning task were more evident at its initial, easier stages. The MSA group showed no consistent evidence of intellectual deterioration as assessed from their performance on subtests of the Wechsler Adult Intelligence Scale (WAIS) and the National Adult Reading Test (NART). Consideration of individual cases showed that there was some heterogeneity in the pattern of deficits in the MSA group, with one patient showing no impairment, even in the face of considerable physical disability.

The results show a distinctive pattern of cognitive deficits, unlike those previously seen using the same tests in patients with Parkinson's and Alzheimer's diseases, and suggesting a prominent frontal-lobe-like component. The implications for concepts of 'subcortical' dementia and 'fronto-striatal' cognitive dysfunction are considered.
\end{abstract}

\section{INTRODUCTION}

In contrast to the extensive investigation of cognitive deficits in Parkinson's disease (PD), Huntington's disease (HD) and progressive supranuclear palsy (PSP) (for a review see Brown and Marsden, 1988), there has not been a single neuropsychological study of multiple system atrophy (MSA), a condition which accounts for 5-10\% of parkinsonians. Multiple system atrophy is a term used to describe a progressive neurological condition, usually incorporating a parkinsonian syndrome (striato-nigral degeneration)

Correspondence to: Dr T. W. Robbins, Department of Experimental Psychology, University of Cambridge, Downing Street, Cambridge CB2 3EB, UK.

(C) Oxford University Press 1992 
and often with additional autonomic failure (Shy-Drager syndrome) or cerebellar and/or pyramidal signs [olivopontocerebellar atrophy (OPCA)] (Berciano, 1982; Oppenheimer, 1983; Quinn, 1989). Multiple system atrophy appears to have distinct neuropathological signs, as shown from the recent findings of glial cytoplasmic inclusions, not only in those regions where cell loss and gliosis are classically found in MSA, such as the putamen, but also in the primary and premotor cerebral cortex and the paracentral gyrus (Papp et al., 1989; Nakazato et al., 1990). As in PD, the brains of patients with MSA show profound depletion of forebrain dopamine, noradrenaline and (more variably) acetylcholine (Spokes et al., 1979).

Cognitive deterioration is not generally considered to be an integral feature of MSA (Quinn, 1989). Although coincidental Alzheimer's disease can occur (Trotter, 1973; Kosaka et al., 1981), this is seen at a rate no higher than in the general age-matched population. The clinical impression is that global cognitive impairment is not a consistent feature of patients with MSA. However, in our experience routine neuropsychological assessment often reveals evidence of underfunctioning of variable and as yet undefined cognitive deficits.

In this study we investigated cognitive function in a group of patients with MSA of the striato-nigral type, concentrating on tests that we have found to be sensitive to frontal lobe damage and PD. In recent papers (Downes et al., 1989; Owen et al., 1991) we introduced an attentional set-shifting paradigm based on learning theory and showed that unmedicated PD patients performed at least as poorly as either medicated patients, who were later in the course of PD, or patients with neurosurgical lesions of the prefrontal cortex. However, patients with temporal lobe excisions or amygdalohippocampectomy are not impaired in this test, showing that the frontal lobe deficits have considerable neural specificity. Another test suitable for assessing frontal lobe function is the Tower of London planning task, which Shallice (1982) showed was sensitive to anterior cortical damage. Owen et al. (1990a) confirmed that performance on a similar computerized version of the test is impaired, in terms of both accuracy and speed measures of thinking, in patients with neurosurgical lesions of the frontal (but not temporal) cortex. The computerized version allowed the inclusion of a motor control condition, where the subject had to make moves defined by the computer program, thus enabling true thinking time on the test problems to be calculated by subtraction. Whereas frontal patients were found to be less accurate on this task, and slower to think about the problems subsequent to the first move (Owen et al., 1990a), patients medicated for PD were no less accurate, but were significantly slower in making their initial move (Morris et al., 1988; Owen et $a l ., 1990 b$, unpublished data). Finally, we employed a test of spatial working memory that has again been found to be sensitive to frontal lobe damage, partly because of the use of inefficient strategies for reducing the load on working memory (Owen et al., 1990 a). Performance on a similar test had previously been found to be normal in PD patients on medication (Morris et al., 1988). Thus, in summary, we employed one test (attentional set-shifting) identifying similar deficits in PD and frontal lobe patients, another (Tower of London) which produced qualitatively different deficits, and a third (spatial working memory) sensitive to frontal lobe damage but not to unmedicated or mild cases of medicated PD.

In order to define the specificity of any deficits found with the tests of frontal lobe function, we also administered, to most of the patients, tests from a battery which we 
had previously shown to be sensitive to memory and learning deficits in PD and Alzheimer's disease (Sahakian et al., 1988).

\section{SUBJECTS}

Multiple system atrophy (MSA) patients

A consecutive series of 16 patients diagnosed as clinically probable MSA of striato-nigral type (MSA-SND) was studied. All patients were diagnosed at the National Hospital for Neurology and Neurosurgery, Queen Square, London, according to the criteria set out by Quinn (1989) (see Table 1). Cases 1-15 had a poor

TABLE 1. CLINICAL FEATURES PRESENT IN MSA GROUP

\begin{tabular}{|c|c|c|c|c|c|c|c|}
\hline Case & Sex & Parkinson & Cerebellar & Pyramidal & Autonomic & $\begin{array}{c}\text { Duration of } \\
\text { disease (yrs) }\end{array}$ & $\begin{array}{c}\text { Hoehn and Yaht } \\
\text { stage }\end{array}$ \\
\hline 1 & $\mathbf{M}$ & + & - & + & + & 9.5 & III \\
\hline 2 & $\mathbf{M}$ & + & - & + & + & 4.5 & IV \\
\hline 3 & $\mathbf{M}$ & + & + & + & + & 7.5 & $\mathrm{~V}$ \\
\hline 4 & $\mathbf{M}$ & + & + & + & + & 2 & III \\
\hline 5 & $\mathbf{M}$ & + & + & + & + & 7 & III \\
\hline 6 & $\mathbf{M}$ & + & + & + & + & 7 & III \\
\hline 7 & $\mathrm{~F}$ & + & - & + & + & 6 & III \\
\hline 8 & $\mathbf{M}$ & + & + & + & + & 9 & $\mathrm{~V}$ \\
\hline 9 & $\mathbf{M}$ & + & + & + & + & 6 & IV \\
\hline 10 & $\mathbf{M}$ & + & + & - & + & 10 & IV \\
\hline 11 & $\mathbf{M}$ & + & - & - & + & 6 & III \\
\hline 12 & $\mathbf{M}$ & + & - & $+1-$ & + & 5.5 & IV \\
\hline 13 & M & + & - & $+1-$ & + & 2.5 & V \\
\hline 14 & $\mathbf{M}$ & + & - & - & + & 10 & III \\
\hline 15 & $\mathbf{M}$ & + & + & - & + & 2 & III \\
\hline 16 & $\mathrm{~F}$ & + & - & - & + & 4 & IV \\
\hline
\end{tabular}

or absent response to levodopa treatment. Case 16 had greater than $50 \%$ response with dyskinesia and fluctuations, but within 2 yrs had developed respiratory stridor, frequent falls, postural faintness and urinary disturbance with abnormal sphincter EMG. The duration of the disease averaged $6 \mathrm{yrs}$, taken from the time of initial symptoms. All but two patients had received a CT scan. One of these two received instead an MRI scan. Six of the patients also underwent $\left[{ }^{18} \mathrm{~F}\right]$ fluoro-dopa PET scans. Only two of the $16 \mathrm{MSA}$ patients were not receiving medication at the time of testing. All of the remainder were receiving levodopa preparations or bromocriptine. Two patients were receiving the anti-cholinergic drug benzhexol (Artane).

\section{Normal controls}

Control subjects were drawn from a large population of volunteers aged between 35 and 70 yrs from London, Cambridgeshire and Newcastle upon Tyne (North-East Age Research Panel). These controls had no history of neurological or psychiatric disorder. From this population, two control groups were selected separately for the Working Memory and Planning (control group $1 ; n=16$ ) and for the Visual Memory batteries (control group 2; $n=15$ ), matched for age and NART IQ. The performance of the MSA group on the ID/ED attentional shift task was evaluated by comparison with a control group ( $n=32$, two for each MSA patient) chosen on a blind basis, but matched overall for age, NART IQ and male:female proportion.

Informed consent was obtained from all patients and controls. 


\section{METHODS}

\section{Clinical neuropsychological assessment}

All patients and controls were tested on the National Adult Reading Test (NART) (Nelson, 1982) to obtain an estimate of their optimal intellectual ability. All but one of the patients received routine clinical assessment in the Department of Psychology, The National Hospital, including a shortened form of the WAIS or WAIS-R (Wechsler, 1955, 1981). Verbal IQs were prorated from the Vocabulary, Similarities, Arithmetic and Digit Span subtests. Performance IQs were prorated from the Picture Completion, Block Design and Picture Arrangement subtests. Similarly, all but one MSA patient received the Recognition Memory Test (Warrington, 1984). Thirteen patients received tests of visual perception (Unconventional Views and Incomplete Letters tests) (Warrington and James, 1967; Warrington and Taylor, 1973). For the McKenna Naming Test (McKenna and Warrington, 1983) scores were available for nine of the 16 patients. Time constraints and physical disability made it impossible for all tests to be administered in the clinical session.

\section{Experimental computerized tests}

The main testing procedures were taken from the Cambridge Neuropsychological Test Automated Battery (CANTAB), a series of cognitive tests run on an Acom BBC Master 128 Microcomputer, with a high resolution Microvitec $30 \mathrm{~cm}$ VDU and a Microvitec Touchtec 501 touch-sensitive screen. These were generally administered in two test sessions, one of approximately $90 \mathrm{~min}$ and one of $45 \mathrm{~min}$. Subjects were seated approximately $0.5 \mathrm{~m}$ from the monitor and it was explained that they would have to respond to stimuli by touching the screen.

They were introduced to the apparatus by way of a 'sensorimotor screening task' in which they were asked to respond to a series of flashing crosses on the screen by placing the index finger of their preferred hand on the centre point of each cross. All subjects satisfactorily completed this task and so were allowed to proceed to the following experimental tests. The first three tests were then taken from the CANTAB Working Memory and Planning tests (see Owen et al., 1990a).

Spatial short-term span. In this computerized version of the Corsi Block Tapping task, the ability of subjects to remember a sequence of squares highlighted on the screen was determined. Each trial began with the same arrangement of nine white $3 \mathrm{~cm}$ squares arranged quasi-randomly in the space of the screen. Subjects were instructed to observe the boxes, as some would change colour one after the other. Their task was to remember the location and the sequential order of the boxes changing colour. During each series, one box would change colour for $3 \mathrm{~s}$ and then return to white before the next in the sequence changed to the same colour. The subject was then prompted by a tone to repeat the sequence by touching the boxes in the same order. During this response sequence, each selected box changed to the same colour for $1 \mathrm{~s}$ and a feedback tone sounded. Following one demonstration trial by the experimenter, the task began at the simplest level of difficulty with a two box sequence. After each successful trial, the number of boxes changing in the next sequence was increased by one to a maximum of nine boxes. After an incorrect attempt at any particular level, an alternative sequence of the same length was presented. This continued until the subject had failed three consecutive trials at any one level. During each trial, a number in the bottom left-hand corner of the screen indicated the length of the current sequence. Also, all boxes changed to the same colour within each series, although on any two adjacent sequences different colours were used to minimize interference. The spatial short-term memory span was calculated as the highest level at which the subject had successfully recalled at least one sequence of boxes. The number of errors made in attaining this level was also recorded.

Spatial working memory task. In this task the subject was required to 'search through' a number of coloured $3 \mathrm{~cm}$ boxes presented on the screen, by touching each one in order to 'open it', thus revealing its contents (see Fig. 1A). The colours of the boxes stayed constant within a trial, but varied over trials. The goal was to collect blue 'tokens' hidden inside the boxes, and once found, to use them to fill an empty column at the side of the screen. The subjects were instructed that at any one time there would be a single token hidden inside one of the boxes. Their task was to search until they found it, at which point the next token would be hidden. The key instruction was that, once a blue token had been found within a particular box, then that box would not be used again to hide a token for that particular trial. Since every box was used once, on each trial the total number of blue tokens to be found corresponded to the number of boxes on 
A

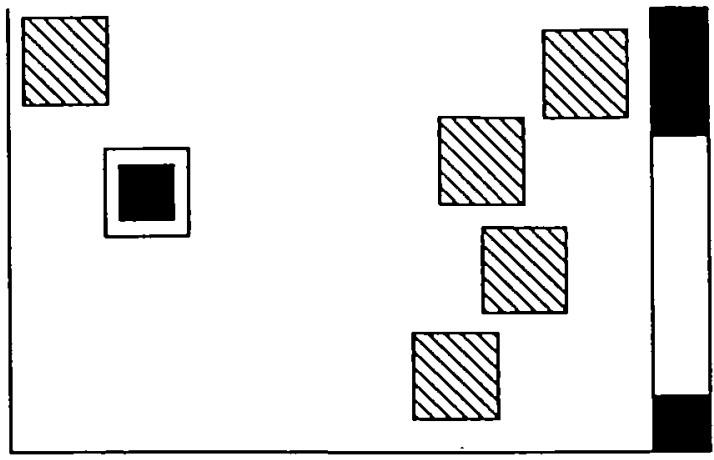

B

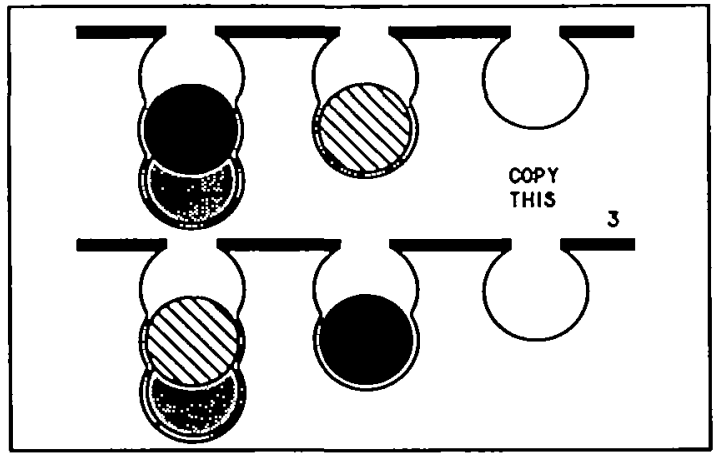

C

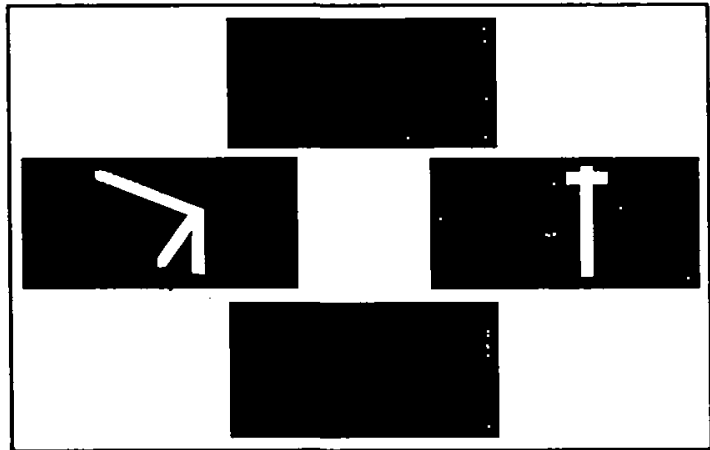

FIG. 1. $A$, the arrangement of boxes on the sceen for the spatial working memory task. The example shows a six box trial, in which one of the boxes has just been chosen to reveal a "blue token' which can now be added to one already in the column on the right-hand side of the screen. This leaves the subject with four more tokens to find, in boxes in which they have not previously been located. B, a sample three move problem for the modified Tower of London task. The goal position of the balls is shown in the top arrangement. $c$, sample pairs of compound stimuli and their arrangement on the screen in the ID/ED attentional set-shifting task.

the screen. In this task, two types of search error are possible. First, a subject may return to open a box in which a blue counter has already been found (a 'between search' error). Secondly, a subject may return to a box already opened in the same search sequence (a 'within search' error). Subjects could search the boxes in any order, but for control purposes, the number of boxes visited (excluding errors), before a 
token was found was determined by the computer. Thus, each subject received the same degree of feedback prior to the first error. After two practice trials with three boxes, there were four test trials with each of four, six and finally, eight boxes. The task was scored according to the number of between and within search errors at each level of difficulty. An efficient strategy for completing this task is to follow a predetermined search sequence, beginning with a particular box and then returning to start a new sequence with that same box as soon as a token has been found. The extent to which this strategy was used was calculated from the number of search sequences starting from the same box, within each of the six and eight box problems. The total of these scores provided a single measure of strategy for each subject, with a high score representing low use of the strategy and a low score representing more extensive usage.

Planning task. This task is closely related to one developed by Shallice and McCarthy, the 'Tower of London', that provides simpler versions of the 'Tower of Hanoi' problem (Shallice, 1982). Two sets of three coloured balls were presented, one in the top half of the screen and one in the bottom half. These were described to the subject as snooker balls since they appeared to be hanging in 'pockets' or 'socks' (see Fig. 18). There were three pockets in each half of the screen, one that could clearly hold three balls, one that could hold two balls, and one that could be completely filled by one ball. On each trial a red ball, a blue ball and a green ball were placed in predetermined positions in the pockets of each of the two displays. The subject was asked to rearrange the balls in the bottom display, such that their positions matched the 'goal' arrangement in the top half of the screen. A ball could be moved by the subject first touching it and then touching an empty position in one of the other pockets. Once selected, a tone sounded and the rim of the ball began to flash, indicating that it was ready to be moved. At any time, the subject could cancel a selected ball by touching it a second time. 'Illegal' moves, such as trying to remove a ball while there was another sitting above it in the same pocket, were carefully explained to the subject and, if attempted, they were registered, but evoked no response from the computer.

The starting position of the balls was varied such that in any particular trial the solution could only be reached after a minimum of two, three, four or five moves. Subjects were instructed to examine the position of the balls at the beginning of each problem and attempt to solve it in the minimum possible number of moves. This was given to them verbally and displayed on the screen throughout each trial. They were encouraged not to make the first move until they were confident that they could execute the entire sequence needed to solve the problem. The maximum moves allowed corresponded to twice the minimum number possible plus one, or plus two, in the case of five-move problems. Following the successful completion of each problem, the VDU displayed the word 'FINISHED', before moving on to the next trial. The program stored the number of moves required by the subject to rearrange the balls and measured the selection and movement latencies for both the first and the subsequent moves. After six practice problems with one and two moves, the subject was given two each of two and three move problems and four each of four and five-move problems. These test problems corresponded exactly to those used in the original Tower of London test.

For each test problem, a 'yoked control'. condition was employed to provide baseline measures of motor initiation and execution times. On each trial of this control condition, the subject was required to follow a sequence of single moves executed by the computer in the top half of the screen by moving the corresponding ball in the lower arrangement. Thus, initially the two arrangements differed by just one ball. Once the subject had made the appropriate move, the top arrangement changed again, so that the subject had to make another single move. The test was 'yoked' to the main test in the sense that in each trial, the movement of the balls was an exact replication of those moved by the subject in the corresponding test trial. The measurement of selection and execution latencies in this control condition provided baseline estimates of motor initiation and execution times.

Test trials and 'yoked' control trials were arranged in four blocks of six problems each. The first six test trials were given (two problems at each of two, three and four moves), followed by their corresponding 'yoked' control trials. Then the remaining six test problems were presented (two at four moves and four at five moves), followed by their yoked control trials. Between each block change there were two practice trials to ensure that the requirements of the current set of tasks had been fully understood.

(i) Accuracy measures of performance. Across the 12 test problems, two measures relating to the number of moves required to reach solution were calculated: (1) the mean number of moves above the minimum possible ('excess moves) provided a general measure of group performance at each level of difficulty; 
(2) the proportion of problems solved in the minimum number of moves ('minimum move solutions') provided more specific information about task difficulty.

(ii) Latency measures of performance. Baseline measures of motor initiation and motor execution times were extracted from the 12 'yoked control' trials. In all cases, latencies were recorded in centiseconds and converted to seconds for the purpose of presentation. The 'motor initiation time' was the mean time between the onset of each problem, and the initiation of the first move (i.e. a correct touch of the required ball). The 'motor execution time' was the time between touching the first ball and completing the sequence of single moves that comprise the whole problem. Since these control problems were yoked to the test problems, the total execution time was divided by the number of moves to provide an estimate of the average movement time per move.

The motor initiation and execution times were used to derive estimates of planning or 'thinking' time in the main task. Two separate estimates were calculated. In each problem, the 'initial thinking time' was the time between the presentation of the problem and the first touch, minus the corresponding motor initiation time, as calculated from the 'yoked control' task. The 'subsequent thinking time' was the time between the selection of the first ball and the completion of the problem minus the total motor execution time from the corresponding control problem. Since this measure clearly varied with problem length, subsequent thinking time scores were divided by the number of moves to give an estimate of the average thinking time 'per move'. In this way, pure estimates of initial and subsequent thinking times were derived, unconfounded by motor initiation or execution times.

Intra- and extra-dimensional (ID/ED) set-shifing paradigm. The test set-up and stimuli were shown to the subjects. Four rectangular boxes, to the top and bottom and to the right and left of centre, appeared on the screen. Two of these contained the test stimuli but the boxes used changed from trial to trial. Subjects were instructed in the following way:

'Now you can see two patterns. One of the patterns is correct. You must point to the one you think is correct. There is a rule you can follow to make sure you make the correct choice each time. The computer will be keeping track of how well you are doing and when it is clear that you know the rule the computer will change it, but this will not happen very often. To begin with, there is nothing on the screen to tell you which of the two patterns is correct, so your first choice will be a simple guess. However, the computer will give a message after each attempt to tell you whether you are right or wrong. You can start now.'

The test then proceeded through a number of stages, each with a different contingency, up to a maximum of nine (see Fig. 1c). For each, continuation to the next stage was dependent on a criterion of six successive correct discriminations being reached. If criterion was not reached at the 50th trial of a stage, then the test was discontinued and subjects did not proceed to the following stage. The order of discrimination was fixed, so that the ED shift always followed the ID shift. However, previous work has established that comparable effects are found when the altemative ordering is used (Roberts et al., 1988). More detailed rationales for the exact design of the test can be found in previously published articles (Roberts et al., 1988; Downes et al., 1989).

To begin with, subjects were given a simple simultaneous discrimination in which the stimuli varied along only one of the two 'dimensions' for deriving the stimuli. These dimensions were purple-filled shapes or white lines. The starting dimension was balanced across subjects and groups. A response to one of the two boxes containing the stimuli resulted in an auditory tone, together with visual feedback which informed the subjects of the correctness of their responses. This feedback was in the form of the words 'correct' and 'wrong' presented respectively in green and red lettering above the middle two boxes. The same feedback was used for each of the subsequent stages. After $1.5 \mathrm{~s}$ the screen cleared and an inter-trial interval of $1 \mathrm{~s}$ was begun.

Following the initial simple discrimination (SD), the remaining eight stages were as follows. For the second stage, simple discrimination reversal (SDR), the discriminanda remained the same, but the previously incorrect choice became the correct one and vice versa, i.e. the contingencies were reversed. At the third stage, compound discrimination (with spatially separated elements) (C-D), the second dimension was introduced with one exemplar of each dimension paired together to form a compound stimulus in two of the response boxes. To succeed, a subject had to continue to respond to the correct exemplar of the previous stage. For this and all subsequent stages, exemplars of the different dimensions were paired in 
a pseudorandom fashion so that all four possible compound stimuli were used, with the constraint that runs of no more than three trials with the same pairings were allowed. The stimuli for the fourth stage, compound discrimination (with elements superimposed) (CD) and subsequent stages were also compounds, but the two exemplars from the different dimensions were superimposed, with the white line always in the foreground. The contingencies were again unchanged from those for the previous two stages. A reversal then occurred at the fifth stage (CDR). New exemplars for both dimensions were introduced at the sixth stage, the intra-dimensional shift (IDS), but the relevant dimension (i.e. shapes or line) was unchanged from Stage 1. This was followed by a further reversal at the seventh stage (IDR). For the penultimate stage, the extra-dimensional shift (EDS), new exemplars were again introduced, but success at this point was dependent on the subject shifting response set to the exemplars of the previously irrelevant dimension. Finally, contingencies were reversed to the previously incorrect exemplar of the new dimension (EDR). The main measure of performance on this task was the stage successfully attained.

Tests of visual memory and learning. It was not possible to administer the complete battery to all of the MSA patients because of the occasional need to limit the length of the test session in order to avoid fatigue. The patients received computerized tests of visual memory and leaming identical to those previously described in detail (Sahakian et al., 1988). These included tests of pattern and spatial recognition $(\mathrm{n}=15)$ simultaneous and delayed matching to sample $(n=13)$ and a conditional visuospatial associative learning task $(\mathbf{n}=11)$. In the pattern recognition test, subjects were shown a number of abstract visual patterns, one after another on the VDU, before being asked to point to which one of two they had previously seen. There were two blocks of 12 patterns to remember. In the spatial recognition test, a white square moved around the VDU screen to five locations in turn. In the test phase, the subjects were asked to point to the locations where they had previously seen the square, in a two-alternative forced choice procedure. The test comprised four blocks of five locations each. In the matching to sample test, subjects were shown a sample abstract visual stimulus on the VDU and were then asked to point to the one which matched exactly this sample from an array of four such stimuli. In the delayed matching-to-sample component, the sample stimulus was turned off before the matching stimuli appeared, either $0 \mathrm{~s}, 4 \mathrm{~s}, 8 \mathrm{~s}$ or $16 \mathrm{~s}$ later. There were five trials in each condition. In the conditional visuospatial associative learning task, subjects were required to remember or learn the locations of abstract visual stimuli in not more than 10 repeated trials. The test began with the requirement to remember the location of only one stimulus, then there were sets of two, three, six and eight stimuli to remember. If the subject failed to reach criterion at any stage, the test was discontinued.

\section{Statistical analysis}

For most of the dependent variables, analysis of variance was used. Where appropriate the data were transformed to meet the assumptions of this method. For most of the test variables, the ANOVA model was a two factor design which included a Between Subjects factor (Group) and a Within Subject factor (e.g. Difficulty Level). Analysis of significant interactions was made by determining the Simple Main Effects (Winer, 1971, pp. 544-545) and with appropriate post hoc comparisons based on the Newman-Keuls test.

For the ID/ED task, the data for the numbers of subjects passing or failing each stage of the test were cast into contingency tables and analysed using the Likelihood Ratio method (Kullback, 1968; Robbins, 1977). The Likelihood Ratio method is particularly useful for analysing data with small cell frequencies and the resulting 'information' statistic (2i) is distributed as $\chi^{2}$ (Kullback, 1968).

The complete control samples were also used to obtain a percentile range of performance on each test for the purpose of assessing the significance of deficits in individual patients.

\section{RESULTS}

\section{Clinical neuropsychological tests}

The MSA patients and controls were matched for age and NART IQ. The mean age and NART estimated IQ for the MSA group was 55.2 (SD = 7.7) yrs and 111.5 $(\mathrm{SD}=8.7)$, respectively. The mean ages of the three control groups were: Group 1 , 54.6 yrs $(\mathrm{SD}=7.3)$; Group $2,56.5$ yrs $(\mathrm{SD}=5.8)$; Group $3,56.6$ yrs $(\mathrm{SD}=5.7)$. 
The respective mean NART estimated IQs for the control groups were $112.6(\mathrm{SD}=6.7)$, $111.7(\mathrm{SD}=7.5)$ and $111.9(\mathrm{SD}=7.0)$. Table 2 shows the characteristics of the individual patients for age, NART IQ, WAIS Verbal and Performance IQs, and

TABLE 2. NEUROPSYCHOLOGICAL CHARACTERISTICS OF MSA GROUP

\begin{tabular}{|c|c|c|c|c|c|c|}
\hline \multirow[b]{2}{*}{ Case } & \multirow[b]{2}{*}{ Age } & \multirow[b]{2}{*}{ NART IQ } & \multirow[b]{2}{*}{ WAIS Verbal IQ } & \multirow[b]{2}{*}{ WAIS Performance IQ } & \multicolumn{2}{|c|}{$\begin{array}{c}\text { Recognition } \\
\text { Memory Tests } \\
\text { (scaled score) }\end{array}$} \\
\hline & & & & & Words & Faces \\
\hline 1 & 61 & 114 & $124^{*}$ & $108^{*}$ & 15 & 12 \\
\hline 2 & 53 & 106 & $99 *$ & $90^{*}$ & 7 & 7 \\
\hline 3 & 54 & 121 & 114 & 116 & 12 & 13 \\
\hline 4 & 54 & 104 & $112^{*}$ & $112^{*}$ & 7 & 9 \\
\hline 5 & 54 & 113 & 113 & 105 & 13 & 13 \\
\hline 6 & 44 & 124 & 126 & 135 & 15 & 17 \\
\hline 7 & 54 & 93 & 93 & 97 & 7 & 7 \\
\hline 8 & 54 & 121 & 127 & 114 & 10 & 8 \\
\hline 9 & 64 & 107 & 107 & 104 & 15 & 13 \\
\hline 10 & 66 & 117 & 103 & 107 & 12 & 3 \\
\hline 11 & 51 & 116 & $102 *$ & $90^{*}$ & 10 & 9 \\
\hline 12 & 59 & 114 & 101 & $\mathrm{nt}$ & 10 & $\mathrm{nt}$ \\
\hline \multirow[t]{2}{*}{13} & 57 & 121 & 122 & 116 & 15 & 16 \\
\hline & 69 & 102 & $\mathrm{nt}$ & $\mathrm{nt}$ & nt & $\mathrm{nt}$ \\
\hline 15 & 50 & 110 & 105 & 120 & 3 & 4 \\
\hline 16 & 39 & 101 & $98 *$ & $99^{*}$ & 13 & 15 \\
\hline
\end{tabular}

Recognition Memory test scores. Only three of the MSA group had a discrepancy between their NART IQ and WAIS Verbal IQ scores of more than 10 points. Thus, the majority of the group showed no evidence of intellectual deterioration. Similarly, in spite of the fact that these patients were motorically impaired, only three had WAIS Verbal Performance discrepancy scores greater than 10 points. No clear pattern of deficits was revealed in the subtest scores. For the Recognition Memory test, on the verbal version one patient was severely impaired and another three showed mild deficits when compared with their expected scores. On the visual version two patients were severely impaired and a further three were mildly deficient. Thus, nine out of 15 patients performed entirely satisfactorily on this test, showing no memory deficits. No patient out of the 13 assessed showed any perceptual deficit. No naming difficulties were evident in the nine patients who were tested on the McKenna Naming test.

\section{Experimental computerized tests}

\section{Spatial span}

There were no significant differences in spatial span between the matched controls and the MSA group $(\mathrm{F}=2.89$, d.f. $=1,30 ; P>0.05)$. The mean spans attained for each group were: Matched Controls, $5.7(S D=1.14)$; $M S A, 5.1(S D=0.93)$. In attaining their highest span score the MSA group averaged only one error and the control group, $0.4(\mathrm{~F}=3.64$, d.f. $=1,30 ; P>0.05)$. 
Tests sensitive to frontal lobe dysfunction

Spatial working memory. Figure 2 shows the mean and SEM number of between search errors at each level of difficulty (four, six and eight boxes) for the control and MSA groups. Analysis of variance showed significant main effects of Group $(F=5.91$,

FIG, 2. Between search errors on the spatial working memory test at different levels of task difficulty for the MSA $(\bullet)$ and matched control $(O)$ group.

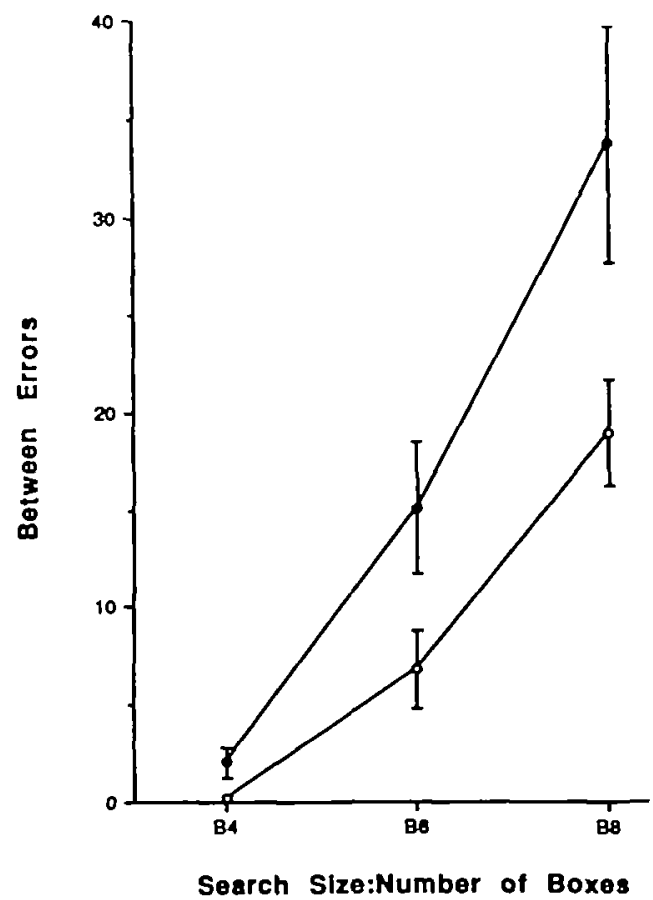

d.f. $=1,30, P=0.02$ ) and Difficulty Level (i.e. number of boxes, four, six or eight) $(\mathrm{F}=54.11$, d.f. $=2,60 ; P<0.001)$. There was also a significant interaction between Group and Difficulty $(\mathrm{F}=3.48$, d.f. $=2,60 ; P<0.05)$. Post hoc analyses of variance at each Difficulty Level showed that the MSA group was significantly worse at the level of eight boxes $(\mathrm{F}=11.1$, d.f. $=1,30 ; P<0.01)$ and the difference at the level of six boxes approached significance $(\mathrm{F}=3.52$, d.f. $=1,30$, ns). Within search errors were variable and at a low level, and so were summed over the levels of difficulty. The control group averaged $3.38(\mathrm{SD}=3.79)$ and the MSA group $10.0(\mathrm{SD}=15.82)$ errors, a non-significant difference $(F<1.0)$.

For the strategy score there was no significant difference between the groups [F $<1.0$, d.f. $=1,30 ; P>0.05$; mean (SD) scores, controls 34.4 (4.08) and MSA $35.56(5.88)]$.

Tower of London. There was a non-significant trend for the MSA patients to be significantly less accurate on the minimum moves measure of accuracy of thinking $(\mathrm{F}=3.63$, d.f. $=1,30 ; P>0.05)$. Mean (SD) values were as follows: controls, 9.3 (1.58); MSA 8.3 (2.2). For the excess moves measure, mean (SD) scores are shown 
in Table 3. Analysis of variance showed no group effect $(F=1.92$, d.f. $=1,30$; $P>0.05$ ). The mean scores for the latency measures are also shown in Table 3 . For initial thinking time, there was no significant group difference at any level of difficulty

TABLE 3. SUMMARY OF MEAN (SD) SCORES ON TOWER OF LONDON PLANNING TEST

\begin{tabular}{|c|c|c|}
\hline & $\begin{array}{c}\text { Control group } \\
(n=16)\end{array}$ & $\begin{array}{c}M S A \\
(n=/ 6)\end{array}$ \\
\hline \multicolumn{3}{|c|}{ Excess moves above the minimum } \\
\hline Two moves & 0 & $0.03(0.13)$ \\
\hline Three moves & $0.25(0.53)$ & $0.50(0.81)$ \\
\hline Four moves & $0.89(0.74)$ & $1.44(0.97)$ \\
\hline Five moves & $1.33(1.41)$ & $1.59(1.52)$ \\
\hline \multicolumn{3}{|c|}{ Initial thinking time(s) } \\
\hline Two moves & $1.49(0.80)$ & $1.80(1.51)$ \\
\hline Three moves & $6.82(4.25)$ & $10.69(8.87)$ \\
\hline Four moves & $9.33(5.16)$ & $10.54(8.03)$ \\
\hline Five moves & $11.57(6.60)$ & $12.81(7.57)$ \\
\hline \multicolumn{3}{|c|}{ Subsequent thinking time(s) } \\
\hline Two moves & $0.47(0.56)$ & $0.69(0.83)$ \\
\hline Three moves & $0.58(0.88)$ & $2.58(3.49)$ \\
\hline Four moves & $2.09(2.78)$ & $4.06(2.58)$ \\
\hline Five moves & $1.49(1.86)$ & $2.45(1.98)$ \\
\hline
\end{tabular}

(i.e. two, three, four or five moves) $(\mathrm{F}=1.63$, d.f. $=1,30 ; P>0.05)$. There was a significant effect of difficulty $(\mathrm{F}=21.67$, d.f. $=3,90 ; P<0.001)$, largely because of the large increase after the easier two move problems. However, there was no significant interaction between Group and Difficulty ( $\mathrm{F}<1.0$, d.f. $=3,90 ; P>0.05$ ).

For subsequent thinking time, there were significant main effects of Group $(\mathrm{F}=8.28$, d.f. $=1,30 ; P<0.01)$ and Difficulty $(\mathrm{F}=8.96$, d.f. $=3,90 ; P<0.001)$, with longer thinking times for MSA patients at the three, four and five move problems (see Fig. 3).

As expected, movement times in the yoked control task differed significantly among groups. The MSA group were significantly slower than the controls; for initial movement time, $\mathrm{F}=4.95$, d.f. $=1,30 ; P<0.05$ : for subsequent movement time, $\mathrm{F}=17.89$, d.f. $=1,30 ; \mathrm{P}<0.001$. The mean values for initial movement time across task difficulty varied from 2.98 to $4.42 \mathrm{~s}$ for the MSA group and 2.08 to $2.38 \mathrm{~s}$ for the control group. Parallel data for subsequent movement time were: MSA, 3.55-4.40 s; control, $1.82-2.28 \mathrm{~s}$.

$I D / E D$ set-shifting paradigm. Figure 4 shows the proportion of subjects passing each stage of the ID/ED shift paradigm. These data were analysed using the Likelihood Ratio method for contingency tables. Approximately $88 \%$ of control subjects passed all stages of the test, whereas only $56 \%$ of the MSA group did so. This difference was significant overall $(2 \mathrm{i}=5.63$, d.f. $=1, P<0.02)$. Further analysis focused on individual stages of the test, taking into account only subjects attempting those stages. This showed that a significant point of failure occurred at the EDS stage in the MSA group. Here, two 
FIG. 3. Subsequent thinking time at two, three, four and five move problems on the Tower of London task. - MSA; $\mathrm{O}$, controls.

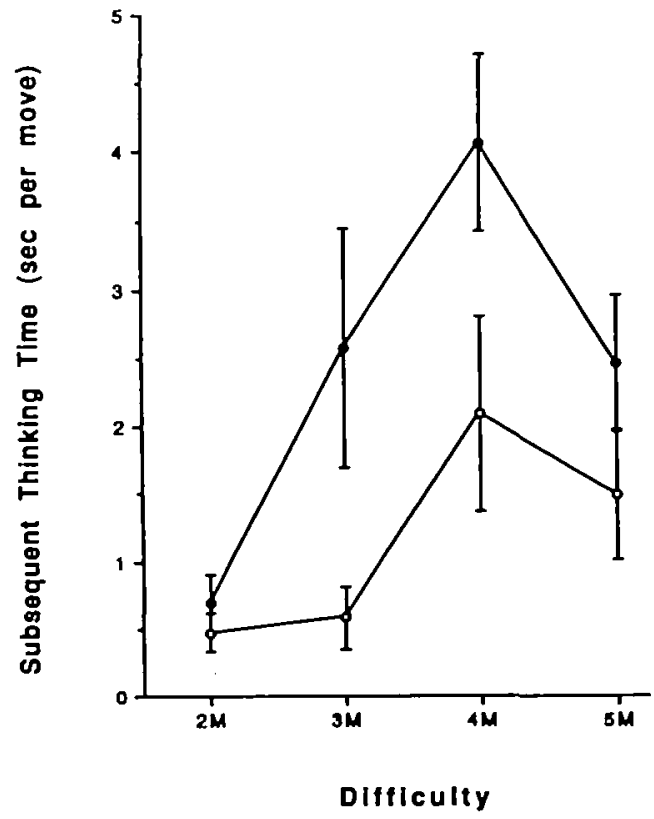

out of $32(6.66 \%)$ of control subjects failed, compared with six out of $15(40 \%)$ of the MSA patients $(2 \mathrm{i}=7.12$; d.f. $=1, P<0.01)$. Thus, the MSA patients exhibited a significant and selective deficit in switching attention between the two dimensions.

Tests of visual memory and learning

The main results are summarized in Table 4.

Pattern and spatial recognition memory. Neither of these tests showed any significant differences between the MSA and control groups.

Simultaneous matching to sample. There was a significant impairment in the MSA group in the simultaneous matching component but not in delayed matching to sample performance, even as a function of delay ( $\mathrm{F}<1.0$, d.f. $=3,78 ; P>0.05$ ).

Conditional visuospatial associative learning. All of the controls successfully learned within 10 trials each set of pattern-location associations, up to and including the final set of eight patterns. In the MSA group, two patients failed to reach criterion at the level of six patterns. For the purposes of analysis of trials and errors to criterion over the entire test, these subjects received scores of 10 (i.e. the maximum score) trials and an error score one more than that of the worst control subject actually attempting the most difficult level. Despite these adjustments, there were no significant differences between the two groups over the test as a whole (see Table 4). Inspection of the data at separate levels of difficulty within the test sequence showed that there were apparent group differences before the most difficult sets of six and eight stimuli. For example, at the easiest levels of the task where the locations of only one, two or three stimuli have to be remembered, only four out of $\mathbf{1 5}$ of the control subjects made any errors 


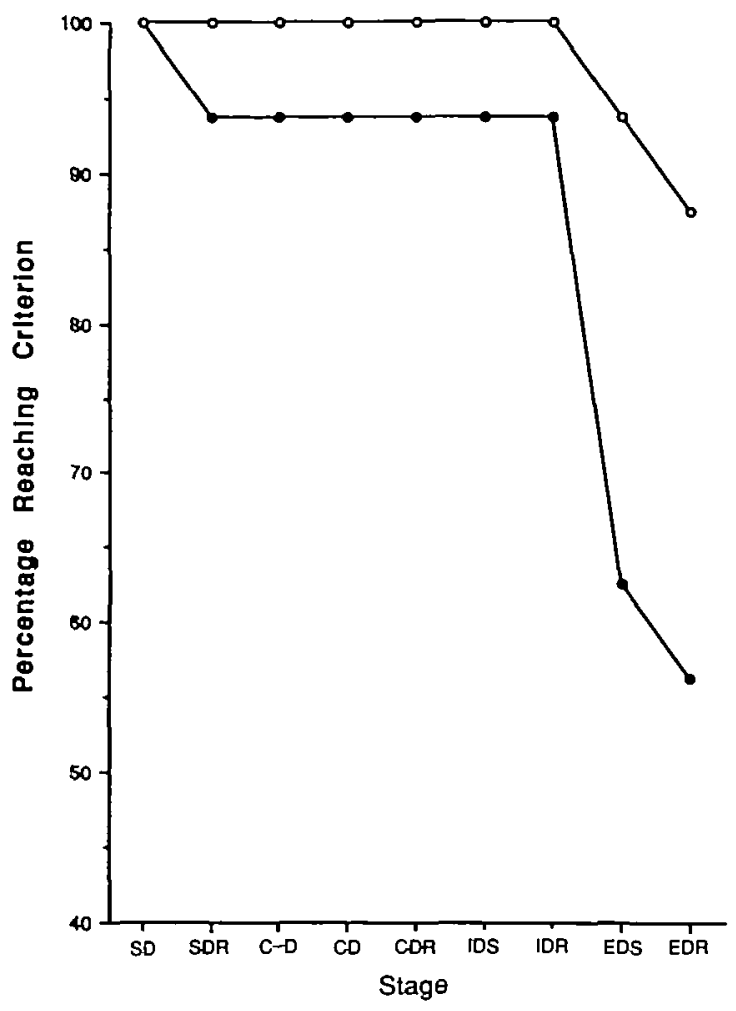

FIG. 4. Cumulative proportion of MSA patients $(\bullet)(n=16)$ and controls $(O)(n=32)$ reaching criterion at various stages of the ID/ED attentional set-shifting paradigm. See text for definitions of each stage.

TABLE 4. SUMMARY OF RESULTS FOR VISUAL MEMORY AND LEARNING BATTERY

MSA group Control group $F$ ratio (d.f.) $P<$

Pattern recognition (maximum 24)

Spatial recognition (maximum 20)

Simultaneous MTS (maximum 5)

Delayed MTS (maximum 5)

$0 \mathrm{~s}$
$4 \mathrm{~s}$
$8 \mathrm{~s}$
$16 \mathrm{~s}$

Overall (maximum 20)

Conditional learning

Total trials to criterion Total errors to criterion
$19.3(4.1)$ 20.3 (2.1)

$\mathrm{F}<1.0(1,28) \mathrm{ns}$

$14.8(2.8)$

$15.8(1.9)$

$F=1.3(1,28) \mathrm{ns}$

$4.4(1.1)$

$5.0(0.0)$

$F=4.5(1,26)$

$P<0.05$
$F=1.3(1,26) \mathrm{ns}$

$\begin{array}{lll}17.1(7.0) & 14.3(3.4) & \mathrm{F}=1.9(1,24) \mathrm{ns} \\ 28.6(26.0) & 21.3(13.7) & \mathrm{F}<1.0(1,24) \mathrm{ns}\end{array}$

Values shown are mean (SD) values. ns $=$ not significant. 
(median 0, range $0-3$ ), whereas nine out of 11 MSA patients committed errors (median 3 , range $2-20$ ). As the distribution of these data violated the assumptions of analysis of variance, the cumulative error scores were compared with a Mann-Whitney U test, which confirmed a significant difference between the groups $(U=23.5, P<0.01)$.

\section{REPRESENTATIVE CASES}

The following four cases (see Table 1) illustrate the range of cognitive performance found in the MSA group. Of these Case 3 was the only one to show no cognitive deficits on any test, Case 9 was the most representative of the group as a whole and Cases 7 and 12 showed atypical patterns of a broader range of impairment.

\section{Case 3}

This administrator developed poor balance, slurred speech and urinary frequency in June 1982. Neurological examination a year later revealed an akinetic-rigid syndrome with a slight tremor of the left arm, Parkinson's disease was diagnosed and Madophar was prescribed with benefit. In November 1983 he developed urinary retention. Prostatectomy was largely unsuccessful, and by a year later he had developed nystagmus and heel-shin ataxia. He was referred to us in January 1989, when, at the age of $59 \mathrm{yrs,} \mathrm{a}$ diagnosis of MSA was made, and he was tested on levodopa medication. His physical condition confined him to a wheel-chair. His CT scan showed generalized atrophy that was, however, greatest in the cerebellum. On neuropsychological testing he had a NART IQ equivalent of 121, a WAIS Verbal IQ of 114 and WAIS Performance IQ of 116 . His visual perceptual skills were normal.

His performance on computerized tests showed a normal spatial span (5). On the spatial working memory task he made only 23 between-search errors, a score at the 75 th percentile for his age and a superior strategy score, in the top $5 \%$ for his age group. On the Tower of London task, his performance was well within the normal range for all measures. He successfully completed the attentional set-shifting task.

He was also most competent on tests of memory. For example, his Warrington Recognition Memory Test scores were in the bright average range. He scored $92 \%$ correct on the pattern recognition and $90 \%$ on the spatial recognition tests of the computerized tests. His performance on the delayed matching to sample test was error free. He successively completed the conditional visuospatial associative learning task with no errors, even at the most difficult level of eight items. This latter performance is exceptional, even for normal healthy subjects of high IQ.

Overall, it is clear that this man exhibited a high degree of intellectual competence, in spite of gross physical disability. He died in July 1990, 18 mths after testing. Post-mortem examination confirmed the clinical diagnosis of MSA.

\section{Case 9}

This shop-owner originally developed nocturia in 1982. One year later, a large prostate was operated on by the supra-pubic route, with good result. However, he then developed slurred speech and began to drag his left foot. He was first seen by a neurologist in December 1986, when a parkinsonian syndrome with left-sided pyramidal signs was found. By February 1988, when he was tested, he had developed additional cerebellar signs and clinically probable MSA was diagnosed. He had a normal CT scan. On neuropsychological testing in February 1988 at age 64 yrs, he had a NART IQ equivalent of 107, a WAIS Verbal IQ of 107 and a Performance IQ of 104. His visuospatial tasks were weak, but within the normal range for his age. His score on the McKenna Naming Test was in the superior range. There was thus little evidence of any generalized intellectual deterioration. When tested he was under levodopa medication. His spatial span was normal (5). He performed at a very low level on the spatial working memory test, making 53 errors at the six box stage and 98 errors at the eight box stage. His strategy score, however, was in the normal range. On the Tower of London test he was only mildly slow in initially thinking about the two move problems, but was otherwise in the normal range. He failed the extra-dimensional shift in the attentional set-shifting paradigm.

His performance on tests of recognition memory was normal. For example, he attained excellent scores of 50/50 (verbal) and 46/50 (visual) on the Warrington Recognition Memory Test and 75\% and 79\% on computerized tests of spatial and pattern recognition memory. He was mildly impaired in reaching criterion 
in paired associates learning, although this deficit was not sustained at the most difficult level (8). Thus at the second test, at level 3 he made five errors, taking three trials, at level 6 he took six trials to reach criterion and made 18 errors, but on the immediately following level (8) he took only three trials in making five errors, a normal level of attainment. Finally, his scores on the matching-to-sample test were as follows. On the simultaneous version of the task and at $0 \mathrm{~s}$ delay he scored consistently $80 \%$, whereas his performance with delays was error free. His performance on this test thus resembled those shown previously by patients with Parkinson's disease (Sahakian et al., 1988). Overall, his performance on the computerized tests is consistent with frontal lobe dysfunction. This deficit is supported by clinical evidence of poor verbal fluency, difficulties with the Similarities subtest of the WAIS and concrete interpretations of proverbs.

\section{Case 7}

This printer's assistant presented in 1982 with falls and postural faintness. A year later, she developed slowness and stiffness and Parkinson's disease was diagnosed. By 1988, additional pyramidal signs were present and a diagnosis of clinically probable MSA was made. Her CT scan revealed brain stem and cerebellar atrophy. She was receiving levodopa and imipramine at the time of testing when her age was $54 \mathrm{yrs}$. Her NART IQ equivalent was 93, her WAIS Verbal IQ 93 and her Performance IQ 97. Her naming skills were consistent with her low average intellectual ability. Her visual perceptual skills were normal. Overall, there was no evidence of generalized intellectual impairment. When tested in March 1988 her spatial span of 5 was in the average range. She scored normally on the test of spatial working memory. On the Tower of London test she was poor on both accuracy and latency measures, particularly at the three move stage and she failed at the extra-dimensional shifting stage of the attentional set-shifting paradigm. Therefore, although this woman was unimpaired on tests of spatial memory storage and spatial working memory, she exhibited deficits in both planning and attentional set-shifting.

On additional testing, she also showed a pattern of mild impairment on tests of visual recognition memory and learning. For example, on the Warrington Recognition Memory tests she scored 41/50 (verbal version) and 40/50 (visual version), these dull average scores being slightly below her predicted optimum level of performance. On computerized tests of spatial and pattern recognition she scored $45 \%$ and $60 \%$, respectively, both scores being significantly below the fifth percentile for control performance. Although she scored $100 \%$ in a test of simultaneous matching to sample, she was impaired on delayed matching to sample at delays of $8 \mathrm{~s}(60 \%)$ and $16 \mathrm{~s}(60 \%)$. She successfully completed a test of visuospatial paired associated learning at the level of 8 , but cumulatively made significantly more errors (34) and took significantly more trials (20) to reach criterion than controls.

In summary, this patient showed deficits in tests sensitive to frontal lobe dysfunction, but was also mildly impaired on tests of recognition memory and learning. The patient died in June 1989; no post mortem was carried out.

\section{Case 12}

This marine engineer had first presented with parkinsonism, diagnosed as Parkinson's disease, in December 1982. However, his response to levodopa therapy was minimal and his disability progressed. He developed several syncopal episodes, his speech became markedly hypophonic, and he developed a disproportional antecollis, but there were no pyramidal or cerebellar signs. A diagnosis of clinically probable MSA was made in June 1986. He was not under medication when tested at age 59 yrs in April 1988. He had a normal CT scan. On neuropsychological testing, his NART IQ equivalent was 114 , giving an estimate of his premorbid intellectual ability within the bright average range. However, his WAIS Verbal IQ was only 101, suggesting mild to moderate intellectual deterioration. The Performance subtests of the WAIS were not administered in view of his physical disability. His visuospatial performance was weak, but within normal limits. Language functions were well preserved.

On the computerized testing, his spatial span was below average (4). For the spatial working memory task his total of 65 between-search errors was below the 10th percentile for his age. He showed no obvious search strategy, his score (47) being worse than any control subject. On the Tower of London he managed only four out of 12 problems in the minimum number of moves. Initial thinking time was significantly lengthened in two, three and four move problems and subsequent thinking time in three move problems. He did manage to complete the attentional set-shifting task.

His performance on memory tests was also somewhat inconsistent. Although performing at an average level on the Recognition Memory test for words and scoring $88 \%$ on the computerized pattern recognition 
test, he was deficient on the spatial form of the test $(55 \%)$ and exhibited a delay-dependent deficit on the test of delayed matching to sample. He failed the conditional visuospatial associative learning task at the level of six items. In summary, despite his normal CT scan, this man showed mild to moderate generalized intellectual deterioration and specific deficits in certain tests of frontal lobe function and visual memory.

\section{DISCUSSION}

In this study we have provided evidence of cognitive dysfunction in patients with MSA, using three tests sensitive to the effects of frontal lobe damage. However, there was no consistent evidence either of deficits in tests of memory and learning or of generalized intellectual deterioration that has been observed in patients with probable dementia of the Alzheimer type (DAT), using the same tests. While some of the deficits seen in the MSA group resembled those we have defined in PD, there were also some differences from the profile of deficits seen in that condition.

Considering the nature of the deficits shown by the MSA group in more detail, there were many similarities to those seen previously after neurosurgical damage to the frontal lobe (Owen et al., 1990a, 1991). For example, the MSA group showed patterns of deficits on the ID/ED set-shifting paradigm, quantitatively and qualitatively similar to those seen in frontal patients. On the spatial working memory test, the MSA group made significantly more between search errors, to a similar degree as following frontal lobe damage, although they did not show the same impairment in strategy seen in that group (Owen et al., 1990a). Their performance on the Tower of London test was also qualitatively similar to that seen in frontal patients. For example, the MSA patients took significantly longer to think about the problems subsequent, but not prior, to the first move of the problems. This lengthened latency was not merely an artefact of the patients' motor impairment, as this was carefully controlled by subtracting the latency to make the same moves used to solve the problems when no planning was required. The MSA group was not significantly impaired on the other main measures of performance on the Tower of London test, although they showed a near significant tendency to show the same lack of accuracy of thinking as displayed by frontal patients. Overall, the pattern of performance of MSA patients on the accuracy and latency measures of the Tower of London test resembles that produced in the milder cases of planning dysfunction following frontal lobe damage. This pattern is markedly different from the one we have defined in PD, where there is strong evidence for lengthening of initial, but not subsequent thinking times on the Tower of London test (Morris et al., 1988), a finding which we have recently replicated with exactly the same version of the test as used here on subgroups of PD patients with relatively severe, as well as moderate, disability (Owen et al., 1990b, unpublished data). Therefore, based on these comparisons, it would appear that, whereas the cognitive deficits seen in PD, MSA and frontal lobe damage do have some gross similarities, the MSA profile is, if anything, more comparable with that seen in the frontal lobe patients than those with PD.

Although the MSA group exhibited deficits in each of the tests of frontal lobe function they did not necessarily show impairment in other cognitive domains such as perception or language. In addition, the deficient performance of the MSA group on the spatial working memory and planning tests could not be attributed to general problems in shortterm memory for spatial information or in terms of the sequencing of responses, because the index of visuospatial span was normal in these patients. This preserved function 
stands in contrast to the reduced spatial span in patients with DAT reported by Sullivan et al. (1986) and also to patients with severe PD studied by us with exactly the same test (Owen et al., 1990b, unpublished data).

In addition, there were no significant differences whatsoever in performance on the computerized tests of spatial and pattern recognition, which are also sensitive to deficits in patients with DAT and medicated PD (Sahakian et al., 1988). This is in agreement with the overall performance on the Warrington Recognition Memory Tests, where only two patients had specific memory deficits and two showed mild impairment (see Table 2). The MSA group were inferior to the matched controls on the other two main tests of the visual memory battery, but in specific and unusual ways. Thus, on the matching-to-sample test they were significantly impaired at the simultaneous stage, where there is no mnemonic component, but showed normal delayed matching performance. This is the exact opposite of the pattern shown by patients early in the course of DAT (Sahakian et al., 1988). The particular pattern of deficit in MSA is also distinct from that reported for PD (Sahakian et al., 1988). Moreover, on the test of visual learning, unlike both patients with DAT and with PD (Sahakian et al., 1988), the MSA patients performed surprisingly well at the more difficult levels of the task, but were inefficient at the earlier stages. Taken together with the deficient performance on the simultaneous component of the matching to sample test, but unimpaired performance on the delay component which immediately follows, this pattern of results is suggestive not of primary learning and memory deficits, but of problems in orientation or attention. These mask the patients' true potential for memory and learning, but can be overcome as the task becomes familiar and attentional resources are gradually marshalled. It is possible that the initial problems are consistent with impairments in attentional functions that depend on executive functions of the frontal lobes (Shallice, 1988).

Although the MSA patients showed a distinctive and significant profile of deficits as a group, there was some heterogeneity of cognitive dysfunction, with one case (Case 3) showing no discernible deficits, even in the face of severe physical disability, but others exhibiting clear impairments on a variety of different tests. The range of cognitive performance present in the MSA group is illustrated by the four case studies. Case 9 showed the most representative profile, with deficient performance on the three main tests sensitive to frontal lobe dysfunction, spatial working memory, attentional set-shifting and the Tower of London planning task, but no evidence of deficits of memory or learning and no signs of generalized intellectual impairment. By contrast, the less typical Cases 7 and 12 showed broader patterns of impairment which included deficits in memory and learning. Indeed, unlike all of the other MSA patients, the deficits in memory and learning for Case 12 resembled those found with the same tests in early cases of DAT (Sahakian et al., 1988). This patient was one of only three who showed a significant NART-Verbal IQ discrepancy (see Table 2), indicating a degree of intellectual deterioration. Therefore, it appears that, in agreement with the clinical impression (Trotter, 1973; Kosaka et al., 1981; Quinn, 1989), there is no compelling evidence of a progressive dementing disorder in MSA.

None of the major deficits in the MSA group were correlated significantly with disease duration or Hoehn and Yahr ratings of severity. The highest correlation found was between Hoehn and Yahr rating and subsequent thinking time at two moves on 
the Tower of London test $(\mathrm{r}=0.45, P>0.05)$. This once more underlines the relative independence of the cognitive from the motor deficits in MSA. Because some of the MSA patients were receiving a variety of drugs at different doses it is more difficult to look for precise associations between medication and cognitive impairment, but none were evident. Although formal ratings of depression were not obtained, it is most unlikely that the pattern of cognitive deficit described here can be explained simply in terms of underlying depression. First, in a recent study of elderly depressed patients (Abas et al., 1990) there were large deficits in the same tests of visual memory and learning that were not apparent in the present MSA group. Secondly, it is difficult to account for the precise pattern of deficits in the MSA group in terms of depression, or indeed fatigue. For example, these patients often performed relatively better at the more difficult stages of the tasks, which is opposite to the pattern generally observed in depression (see review by Robbins et al., 1992).

Ultimately, the profile of cognitive deficit in MSA must be explained in terms of the neural systems that are damaged in this condition. Despite the impairments in tests sensitive to frontal lobe dysfunction and the deficits in memory and learning in some patients seen here, the literature has reported no clear evidence of atrophy or observable changes in the cerebral cortex or hippocampus. In the forebrain, the main signs are striatal DA loss and degeneration of the striatum itself (Spokes et al., 1979; Oppenheimer, 1983). Some studies employing neuro-imaging have demonstrated a relatively lower caudate uptake of $\left[{ }^{18} \mathrm{~F}\right]$ fluoro-dopa in MSA patients, in contrast to the relatively preserved uptake in this region in PD (Nahmias et al., 1985; Rutledge et al., 1987; Brooks et al., 1990a,b).

Therefore, one obvious possibility is that the cognitive deficits seen in MSA are caused by striatal pathology, disturbing the neural circuitry intimately linking the frontal cortex to the striatum (Alexander et al., 1986; Taylor et al., 1986). An alternative, though not mutually exclusive, hypothesis is that the deficits do indeed depend upon the dysfunction of other forebrain structures that results from neurochemical pathology. For example, Spokes et al. (1979) report from four cases, clear evidence of disturbances in glutamatergic neurons in the hippocampus, and in the only region of neocortex assayed, the motor cortex. In addition, the cognitive deficit in MSA might also reflect deafferentation of the neocortex and other forebrain structures of chemically defined pathways originating in the 'isodendritic core' (Agid et al., 1987). For example, there is evidence of noradrenergic depletion in the locus coeruleus and a number of forebrain regions, consistent with degeneration of the coeruleal cortical noradrenergic pathway (Spokes et al., 1979). Moreover, as in both PD and DAT (Agid et al., 1987) there is evidence of cholinergic deficits in the hippocampus, although in the four cases of MSA reported by Spokes et al. (1979) the reduction in hippocampal choline acetyltransferase activity varied from about $45 \%$ to $95 \%$. Such observations have interesting implications for some of the individual data reported here, where, for example, visual memory and learning were also variably affected (Cases 7 and 12).

The pattern of deficits shown in MSA as impaired set-shifting on the ID/ED paradigm, deficient spatial working memory and slowness in thinking (or bradyphrenia) is perhaps comparable with the cluster of deficits sometimes called 'subcortical' as distinct from 'cortical' dementia (Albert et al., 1974). The former syndrome has been suggested to subsume the cognitive and behavioural deficits shown by patients with pathology of 
the basal ganglia and other subcortical structures, including PD, HD and PSP, whereas the latter is more usually associated with DAT. On the basis of the present results, there is certainly some evidence to support a dichotomy between the cognitive profile in early DAT on the one hand and MSA, PD and HD on the other. For example, whereas patients with each of the latter disorders are impaired on the ID/ED attentional set-shifting task (Downes et al., 1989, and unpublished results), no such deficit is associated with mild cases of DAT despite much more severe deficits in visual leaming and memory (Sahakian et al., 1990). However, the concept of subcortical dementia has been criticized on the grounds of the similarity of the cognitive profile to that produced by frontal lobe damage, resulting in the suggestion of a more appropriate term 'fronto-subcortical dementia' (see Cummings, 1986; Brown and Marsden, 1988). The comparison we have made between frontal lobe patients and the MSA group is clearly directly relevant to this issue. It does appear that the MSA syndrome is comparable with the effects of frontal lobe damage in many aspects, supporting the possibility of a 'fronto-subcortical' or perhaps more specifically, 'fronto-striatal' syndrome. However, a further comparison of interest is with the cognitive profile in $\mathrm{PD}$, which appears distinct from that of MSA, both in terms of the breadth of cognitive deficits seen in the later stages of PD, and in terms of the qualitative nature of the deficits we have described above for the tests sensitive to frontal lobe dysfunction. These differences clearly warrant more detailed exploration, but their existence suggests that the syndrome of 'subcortical' or 'fronto-subcortical' dementia may well not be unitary in nature.

\section{ACKNOWLEDGEMENTS}

We wish to thank Professor E. K. Warrington and the staff of the Department of Psychology, The National Hospital, for advice and help throughout the course of this study. This work was supported by a Major Award from the Wellcome Trust.

\section{REFERENCES}

Abas MA, Sahakian BJ, Levy R (1990) Neuropsychological deficits and CT scan changes in elderly depressives. Psychological Medicine, 20, 507-520.

Agid Y, RUberg M, Dubois B, Pillon B (1987) Anatomoclinical and biochemical concepts of subcortical dementia. In: Cognitive Neurochemistry. Edited by S. M. Stahl, S. D. Iversen and E. C. Goodman. Oxford: Oxford University Press, pp. 248-271.

Albert ML, Feldman RG, Willis AL (1974) The 'subcortical dementia' of progressive supranuclear palsy. Joumal of Neurology, Neurosurgery, and Psychiatry, 37, 121-130.

Alexander GE, DeLong MR, Strick PL (1986) Parallel organization of functionally segregated circuits linking basal ganglia and cortex. Annual Review of Neuroscience, 9, 357-381.

BERCIANo J (1982) Olivopontocerebellar atrophy: a review of 117 cases. Journal of the Neurological Sciences, 53, 253-272.

Brooks DJ, Ibanez V, SAwle GV, Quinn N, Lees AJ, Mathias CJ et al. (1990a) Differing patterns of striatal ${ }^{15} \mathrm{~F}$-dopa uptake in Parkinson's disease, multiple system atrophy, and progressive supranuclear palsy. Annals of Neurology, 28, 547-555.

Brooks DJ, Salmon EP, Mathias CJ, Quinn N, Leenders KL, Bannister R el al. (1990b) The relationship between locomotor disability, autonomic dysfunction, and the integrity of the striatal dopaminergic system in patients with multiple system atrophy, pure autonomic failure, and Parkinson's disease, studied with PET. Brain, 113, 1539-1552.

Brown RG, MARSDEN CD (1988) 'Subcortical dementia': the neuropsychological evidence. Neuroscience, 25, 363-387. 
Crawford JR, Allan KM, Besson JaO, Cochrane RHB, Stewart LE (1990) A comparison of the WAIS and WAIS-R in matched U.K. samples. British Joumal of Clinical Psychology, 29, $105-109$.

Cummings JL (1986) Subcortical dementia: neuropsychology, neuropsychiatry, and pathophysiology. British Journal of Psychiatry, 149, 682-697.

Downes JJ, Roberts AC, Sahakian BJ, Evenden JL, Morris RG, Robbins TW (1989) Impaired extradimensional shift performance in medicated and unmedicated Parkinson's disease: evidence for a specific attentional dysfunction. Neuropsychologia, 27, 1329-1343.

Kosaka K, Issuka R, Mizutani Y, Kondo T, Nagatsu T (1981) Striatonigral degeneration combined with Alzheimer's disease. Acta Neuropathologica, Berlin, 54, 253-256.

KULlBACK S (1968) Information Theory and Statistics. New York: Dover.

MCKenna P, WARrIngton EK (1983) The Graded Naming Test. Windsor, Berkshire: NFER-Nelson.

Morris RG, Downes JJ, Sahakian BJ, Evenden JL, Heald A, Robbins TW (1988) Planning and spatial working memory in Parkinson's disease. Journal of Neurology, Neurosurgery, and Psychiatry. 51, $757-766$.

Nahmias C, Garnett ES, Firnau G, LaNG A (1985) Striatal dopamine distribution in parkinsonian patients during life. Journal of the Neurological Sciences, 69, 223-230.

Nakazato Y, Yamazaki H, Hirato J, Ishida Y, Yamaguchi H (1990) Oligodendroglial microtubular tangles in olivopontocerebellar atrophy. Joumal of Neuropathology and Experimental Neurology, 49, $521-530$.

Nelson HE (1982) National Adult Reading Test (NART): for the Assessment of Premorbid Intelligence in Patients with Dementia. Windsor, Berkshire: NFER-Nelson.

OPPENHEIMER D (1983) Neuropathology of progressive autonomic failure. In: Autonomic Failure: $A$ Textbook of Clinical Disorders of the Autonomic Nervous System. Edited by R. Bannister. Oxford: Oxford University Press, pp. 267-283.

OWen AM, Downes JJ, Sahakian BJ, Polkey CE, Robbins TW (1990a) Planning and spatial working memory following frontal lobe lesions in man. Neuropsychologia, 28, 1021-1034.

Owen AM, Galton M, Leigh PN, Marsden CD, Quinn NP, Sahakian BJ et al. (1990b) Planning and spatial working memory in Parkinson's disease. Society for Neuroscience Abstracts, $16,1109$.

OWen AM, Roberts AC, Polkey CE, Sahakian BJ, Robbins TW (1991) Extra-dimensional versus intradimensional set shifting performance following frontal lobe excisions, temporal lobe excisions or amygdalo-hippocampectomy in man. Neuropsychologia, 29, 993-1006.

PAPP MI, KAHN JE, LANTOS PL (1989) Glial cytoplasmic inclusions in the CNS of patients with multiple system atrophy (striatonigral degeneration, olivopontocerebellar atrophy and Shy-Drager syndrome). Journal of the Neurological Sciences, 94, 79-100.

QUINN N (1989) Multiple system atrophy - the nature of the beast. Journal of Neurology, Neurosurgery, and Psychiatry, 52, Special Supplement, 78-89.

RoBBins TW (1977) A critique of the methods available for the measurement of spontaneous motor activity. In: Handbook of Psychopharmacology, Volume 7. Edited by L. L. Iversen, S. D. Iversen and S. H. Snyder. New York and London: Plenum Press, pp. 37-82.

RobBins TW, JoYce EM, SAHAKIAN BJ (1992) The neuropsychology and neuroimaging of affective disorders. In: Handbook of Affective Disorders. Edited by E. S. Paykel. London: Churchill Livingstone. In press.

RoberTS AC, RobBins TW, EverITt BJ (1988) The effects of intradimensional and extradimensional shifts on visual discrimination learning in humans and non-human primates. Quarterly Joumal of Experimental Psychology, B, 40,321-341.

Rutledge JN, Hilal SK, Silver AJ, Defendini R, Fahn S (1987) Study of movement disorders and brain iron by MR. AJNR: American Journal of Neuroradiology, 8, 387-411.

Sahakian BJ, Morris RG, Evenden Jl, Heald A, Levy R, Philfot M et al. (1988) A comparative study of visuospatial memory and learning in Alzheimer-type dementia and Parkinson's disease. Brain, $111,695-718$.

Sahakian BJ, Downes JJ, Eagger S, Evenden JL, Levy R, Philfot MP et al. (1990) Sparing of attentional relative to mnemonic function in a subgroup of patients with dementia of the Alzheimer type. Neuropsychologia, 28, $1197-1213$.

Shallice T (1982) Specific impairments of planning. Philosophical Transactions of the Royal Society of London, B, 298, 199-209. 
SHALLICE T (1988) The allocation of processing resources: higher-level control. In: From Neuropsychology to Mental Structure. Edited by T. Shallice. Cambridge: Cambridge University Press, pp. 328-352. SPOKEs EGS, BANNISTER R, OPPENHeImer DR (1979) Multiple system atrophy with autonomic failure. Journal of the Neurological Sciences, 43, 59-82.

Sullivan EV, Corkin S, Growdon JH (1986) Verbal and nonverbal short-term memory in patients with Alzheimer's disease and in healthy elderly subjects. Developmental Neuropsychology, 2, 387-400.

TAYLOR AE, SAINT-Cyr JA, LANG AE (1986) Frontal lobe dysfunction in Parkinson's disease: the cortical focus of neostriatal outflow. Brain, 109, 845-883.

Trotter JL (1973) Striatonigral degeneration, Alzheimer's disease, and inflammatory changes. Neurology, Minneapolis, 23, 1211-1216.

WARRINGTON EK (1984) Recognition Memory Test. Windsor, Berkshire: NFER-Nelson.

WARRINGTON EK, JAMES M (1967) Disorders of visual perception in patients with localised cerebral lesions. Neuropsychologia, 5, 253-266.

WARRINGTON EK, TAYLOR A (1973) The contribution of the right parietal lobe to object recognition. Cortex, $9,152-164$.

WeChSLER D (1955) Wechsler Adult Intelligence Scale. New York: Psychological Corporation.

WeChSLeR D (1981) The Wechsler Adult Intelligence Scale-Revised. San Antonio, TE: Psychological Corporation.

WINER BJ (1971) Statistical Principles in Experimental Design. Second edition. New York: McGraw-Hill.

(Received June 6, 1991. Accepted October 19, 1991) 